\title{
Avaliação dos casos suspeitos de infecção do SARS-CoV-2 em crianças e em adolescentes de um hospital do Brasil
}

\author{
Evaluation of suspected cases of SARS-CoV-2 infection in children and adolescents in a hospital in
} Brazil

Evaluación de casos sospechosos de infección por SARS-CoV-2 en niños y adolescentes en un hospital de Brasil

\section{Resumo}

A COVID-19 consiste na doença causada pela infecção do vírus SARS-CoV-2. A população pediátrica, corresponde de 1 a $5 \%$ dos casos confirmados da doença, variando de acordo com o país em estudo. Este trabalho tem como objetivo analisar os casos infantojuvenis suspeitos e confirmados de COVID-19 em um hospital de Sergipe. Trata-se de um estudo observacional transversal envolvendo 1.388 pacientes, menores de 20 anos, durante março de 2020 e setembro de 2021. Entre os pacientes, 54,8\% eram do sexo masculino, 10,4\% apresentavam alguma comorbidades. Observou-se que a dispneia estava relacionada com a faixa etária, sendo frequente em crianças. Além disso, algumas manifestações clínicas foram associadas a infecção pelo SARS-CoV-2, como cefaleia $(\mathrm{p}=0,021)$, diarreia $(\mathrm{p}=0,002)$, desconforto respiratório $(\mathrm{p}<0,001)$, dispneia $(\mathrm{p}<0,001)$, espirro $(\mathrm{p}<0,001)$ e saturação de $\mathrm{O}_{2}<95 \%(\mathrm{p}<0,001)$. Houve relação entre os pacientes que evoluíram para óbito com algumas variáveis como presença de comorbidades, desconforto respiratório, espirro, necessidade de estabilização e UTI ( $\mathrm{p}<0,001$ ). A maioria dos casos pediátricos são assintomáticos ou leves, além disso as crianças apresentam melhor desfecho na COVID-19, visto que um pequeno percentual evolui para óbito, geralmente associado com a presença de comorbidades pré-hospitalares.

Palavras-chave: COVID-19; Infecções respiratórias; Criança; Adolescente; Diagnóstico.

\begin{abstract}
A COVID-19 is a disease caused by infection with the SARS-CoV-2 virus. The pediatric population corresponds to 1 to $5 \%$ of confirmed cases of the disease, varying according to the country under study. This work aims to analyze suspected and confirmed cases of children and adolescents of COVID-19 in a hospital in Sergipe. This is a crosssectional observational study involving 1,388 patients, under 20 years of age, during March 2020 and September
\end{abstract}


2021. Among patients, $54.8 \%$ were male, $10.4 \%$ had some comorbidities. It was observed that dyspnea was related to age, being frequent in children. In addition, some clinical manifestations were associated with SARS-CoV-2 infection, such as headache $(\mathrm{p}=0.021)$, diarrhea $(\mathrm{p}=0.002)$, respiratory distress $(\mathrm{p}<0.001)$, dyspnea $(\mathrm{p}<0.001)$, sneezing $(\mathrm{p}$ $<0.001)$ and $\mathrm{O} 2$ saturation $<95 \%(\mathrm{p}<0.001)$. There was a relationship between patients who progressed to death and some variables such as the presence of comorbidities, respiratory distress, sneezing, need for stabilization and ICU $(\mathrm{p}<0.001)$. Most pediatric cases are asymptomatic or mild, and children have a better outcome in COVID-19, as a small percentage evolves to death, usually associated with the presence of pre-hospital comorbidities.

Keywords: COVID-19; Respiratory tract infections; Child; Adolescent; Diagnosis.

\section{Resumen}

COVID-19 es una enfermedad causada por una infección con el virus SARS-CoV-2. La población pediátrica corresponde al 1 al 5\% de los casos confirmados de la enfermedad, variando según el país en estudio. Este trabajo tiene como objetivo analizar casos sospechosos y confirmados de niños y adolescentes de COVID-19 en un hospital de Sergipe. Se trata de un estudio observacional transversal en el que participaron 1.388 pacientes, menores de 20 años, durante marzo de 2020 y septiembre de 2021. Entre los pacientes, el 54,8\% eran varones, el 10,4\% presentaba alguna comorbilidad. Se observó que la disnea se relacionó con la edad, siendo frecuente en niños. Además, algunas manifestaciones clínicas se asociaron con la infección por SARS-CoV-2, como dolor de cabeza $(\mathrm{p}=0,021)$, diarrea (p $=0,002)$, dificultad respiratoria $(\mathrm{p}<0,001)$, disnea $(\mathrm{p}<0,001)$, estornudos $(\mathrm{p}<0,001)$ y saturación de $02<95 \%(\mathrm{p}$ $<0,001)$. Hubo relación entre los pacientes que progresaron a muerte y algunas variables como la presencia de comorbilidades, dificultad respiratoria, estornudos, necesidad de estabilización y UCI ( p <0,001). La mayoría de los casos pediátricos son asintomáticos o leves, y los niños tienen una mejor evolución en el COVID-19, ya que un pequeño porcentaje evoluciona a la muerte, generalmente asociado a la presencia de comorbilidades prehospitalarias. Palabras clave: COVID-19; Infecciones de las vías respiratorias; Niño; Adolescente; Diagnóstico.

\section{Introdução}

A COVID-19 consiste na doença causada pela infecção do vírus Síndrome Respiratória Aguda Grave Coronavírus 2 (SARS-CoV-2), um novo tipo de coronavírus identificado em dezembro de 2019 na China. Desde março de 2020, foi declarada a pandemia de COVID-19 pela Organização Mundial da Saúde (OMS). A população pediátrica, que abrange do 0 aos 19 anos de acordo com OMS e Ministério da Saúde (MS), corresponde de 1 a 5\% dos casos confirmados da doença, variando de acordo com o país em estudo, além de que nessa faixa etária a incidência da doença aumenta com a idade (Martins et al., 2021). E, embora não sejam o grupo mais afetado pela COVID-19, a faixa pediátrica também sofre tanto com as manifestações biológicas, quanto socialmente e psicologicamente devido às condições impostas pela pandemia como, por exemplo, o isolamento social (Aydogdu, 2020; Toso et al., 2020).

Dentre as manifestações que as crianças e os adolescentes apresentam durante a infecção pelo SARS-CoV-2, tem-se a febre e a tosse como as mais comuns e, no aspecto radiológico, a opacidade bilateral em vidro fosco como a alteração mais observada (Qju et al, 2020; Lu et al, 2020). Em casos mais graves, o paciente pode apresentar pneumonia ou síndrome respiratória aguda grave (SRAG) devido à infecção (Martins et al, 2021; Hillesheim et al, 2020). Em 2020, da Semana Epidemiológica 1 à 38, foram notificadas 9.483 hospitalizações por Síndrome Respiratória Aguda Grave (SRAG) em pacientes com COVID-19 de 0 a 19 anos, o que corresponde a 2,4\% de todas as hospitalizações de SRAG por COVID-19 desse período. Ademais, de tais hospitalizações, as crianças e adolescentes representam $0,68 \%$ dos casos que tiveram o óbito como desfecho (Brasil, 2020).

Nos Estados Unidos, em locais com baixas taxas de vacinação, foi observado um aumento do número de hospitalizações infantojuvenil associada à predominância da variante Delta do vírus no país, com maior necessidade de cuidados intensivos (Deville et al, 2021). Ademais, sabe-se que o diagnóstico diferencial da infecção pelo SARS-CoV-2 é difícil na pediatria, visto que há diversas doenças pediátricas comuns, como infecções pelo Rinovírus, Influenza A e B e Vírus Sincicial Respiratório, que apresentam manifestações clínicas similares às da COVID-19, por isso a necessidade de exames laboratoriais etiológicos para confirmar o agente responsável pelo quadro (Ramos et al, 2020; Lima et al, 2021). No que diz respeito ao tratamento da doença nessa população, baseia-se no manejo sintomático de acordo com a gravidade do quadro 
clínico (Deville et al, 2021).

Este trabalho tem como objetivo avaliar o aspecto epidemiológico da população pediátrica com quadro suspeito e confirmado de COVID-19 em Sergipe para, desse modo, contribuir com a base de dados dessa doença tão recente, sobretudo, nesse grupo, uma vez que sua prevalência é menor quando comparada a pacientes adultos ou idosos.

\section{Metodologia}

Trata-se de um estudo observacional analítico transversal quantitativo realizado entre 24 de março de 2020 e 15 de setembro de 2021 na Associação Aracajuana de Beneficência (Hospital e Maternidade Santa Isabel) em Aracaju (Sergipe), a qual é referência para cirurgia geral, pediatria, atenção ao parto e ao nascimento. O hospital contém, atualmente, 7 leitos de Unidade de Terapia Intensiva pediátrica (UTI), 20 leitos de Unidade de Terapia Intensiva Neonatal (UTIN), 20 leitos de Unidade de Cuidado Neonatal Convencional (UCINCo), além disso a Unidade de Urgência e Emergência Pediátrica local realiza cerca de 3.500 atendimentos mensais.

Neste estudo foi incluído os pacientes menores de 20 anos, de ambos os sexos, do estado de Sergipe, que realizaram exame laboratorial para detecção do SARS-CoV-2. Enquanto que se excluiu os casos em que o prontuário não continha informações necessárias para atingir os objetivos propostos. Ressalta-se que a definição de caso suspeito e confirmado de COVID-19 atende ao exposto nas Diretrizes para diagnóstico e tratamento da COVID-19 do Ministério da Saúde. Em que caso suspeito era definido como indivíduo com quadro respiratório agudo, caracterizado por sensação febril ou febre, mesmo que relatada, acompanhada de tosse ou dor de garganta ou coriza ou dificuldade respiratória ou obstrução nasal, enquanto que caso confirmado seria caso suspeito com laboratório confirmado (biologia molecular ou imunológico) ou clínico-epidemiológico.

Para coleta de dados dos pacientes com suspeita de infecção por SARS-CoV-2 foi utilizado uma ficha padronizada para extrair as informações necessárias do Serviço de Controle de Infecção Hospitalar. Avaliou-se os dados demográficos, os achados clínicos e laboratoriais, a conduta terapêutica e o desfecho clínico de 1.388 casos suspeitos de COVID-19 em crianças e em adolescentes. Em que as crianças foram definidas pela faixa etária de 0 a 12 anos incompleto e adolescentes de 12 anos completos até 19 anos incompletos, de acordo com o MS.

As variáveis categóricas foram descritas por meio de frequência absoluta e relativa percentual e a hipótese de independência entre essas variáveis foi testada por meio dos testes Qui-Quadrado de Pearson ou Exato de Fisher. Já as variáveis contínuas foram descritas através de média, mediana e desvio padrão, sua hipótese de aderência e distribuição normal foi testada por meio do teste de Shapiro-Wilks. Como não confirmada, a hipótese de igualdade das medidas de tendência central foi testada por meio do teste T de Mann-Whitney. O nível de significância adotado foi de 5\% e o software utilizado foi o R Core Team 2021 (Versão 4.1.0).

Para início dessa pesquisa foi necessário a aprovação do Comitê de Ética e Pesquisa (CEP) da Universidade Federal de Sergipe, CAAE: 34816620.8.0000.5546. Ademais, todos os processos realizados estavam de acordo com Resolução $\mathrm{n}^{\circ}$ 150, de 7 de abril de 2019, a Resolução Conselho Nacional de Saúde (CNS) nº 510 de 2016, a Resolução CNS no 466 de 2012 e a Norma Operacional n 001 de 2013 do CNS e suas complementares. Outrossim, como trata-se de um estudo realizado por meio da consulta na base de dados do Hospital, o Termo de Consentimento Livre e Esclarecido (TCLE) foi dispensado pelo CEP.

\section{Resultados}

No total, foram atendidos 1.388 casos suspeitos de infecção pelo SARS-CoV-2 durante o período desse estudo, sendo que a mediana da idade desses pacientes foi de 0,87 anos (IIQ = 0,08-4,00), em que $1.294(93,2 \%)$ eram crianças e $94(6,8 \%)$ adolescentes. Além disso, 761 (54,8\%) pacientes eram do sexo masculino e $145(10,4 \%)$ apresentavam alguma comorbidades subjacentes, em que as mais frequentes foram as doenças respiratórias. O diagnóstico de COVID-19 foi confirmado em 399 
$(28,7 \%)$ casos, dos casos descartados, 5 pacientes $(0,4 \%)$ tiveram diagnóstico etiológico de infecção pelo vírus Influenza, enquanto 1.003 casos $(72,3 \%)$ por outras etiologias (Tabela 1$)$.

Tabela 1: Características dos casos suspeitos de infecção pelo SARS-CoV-2 em um hospital de Sergipe.

\begin{tabular}{|c|c|c|c|c|c|}
\hline & $\mathbf{n}$ & $\%$ & \% Válido & Mediana & IIQ \\
\hline Idade & & & & 0,87 & $0,08-4,00$ \\
\hline \multicolumn{6}{|l|}{ Faixa etária } \\
\hline Criança & 1294 & 93,2 & & & \\
\hline Adolescente & 94 & 6,8 & & & \\
\hline \multicolumn{6}{|l|}{ Sexo } \\
\hline Feminino & 627 & 45,2 & & & \\
\hline Masculino & 761 & 54,8 & & & \\
\hline \multicolumn{6}{|l|}{ Procedência } \\
\hline Capital & 1169 & 84,2 & & & \\
\hline Interior & 214 & 15,4 & & & \\
\hline Comorbidades & 145 & 10,4 & & & \\
\hline HAS & 61 & 4,4 & 42,1 & & \\
\hline Doença Cardiovascular & 12 & 0,9 & 8,3 & & \\
\hline Desnutrição & 3 & 0,2 & 2,1 & & \\
\hline Doenças cromossômicas & 5 & 0,4 & 3,4 & & \\
\hline Doença Renal & 2 & 0,1 & 1,4 & & \\
\hline Imunossupressão & 6 & 0,4 & 4,1 & & \\
\hline Doença Metabólica & 9 & 0,6 & 6,2 & & \\
\hline Doença Neurológica & 13 & 0,9 & 9,0 & & \\
\hline Síndrome Genética & 2 & 0,1 & 1,4 & & \\
\hline Doença Respiratória & 79 & 5,7 & 54,5 & & \\
\hline Gestante de Risco & 4 & 0,3 & 2,8 & & \\
\hline Laringomalacia & 2 & 0,1 & 1,4 & & \\
\hline Obesidade & 4 & 0,3 & 2,8 & & \\
\hline Outras Síndromes & 3 & 0,2 & 2,1 & & \\
\hline PO de abdome obstrutivo & 1 & 0,1 & 0,7 & & \\
\hline Prematuridade & 5 & 0,4 & 3,4 & & \\
\hline Tumor & 1 & 0,1 & 0,7 & & \\
\hline \multicolumn{6}{|l|}{ Condição do Paciente } \\
\hline Suspeito & 182 & 13,1 & & & \\
\hline Confirmado & 399 & 28,7 & & & \\
\hline Descartado & 807 & 58,1 & & & \\
\hline \multicolumn{6}{|l|}{ Diagnóstico } \\
\hline COVID19 & 380 & 27,4 & & & \\
\hline Influenza & 5 & 0,4 & & & \\
\hline Outros & 1003 & 72,3 & & & \\
\hline PCR Detectável & 761 & 54,8 & 99,0 & & \\
\hline \multicolumn{6}{|l|}{ Tipo de Leito } \\
\hline Clínico & 999 & 72,0 & & & \\
\hline UTI & 164 & 11,8 & & & \\
\hline Estabilização & 45 & 3,2 & & & \\
\hline Ventilação Mecânica & 68 & 4,9 & & & \\
\hline Sintomático & 1369 & 98,6 & & & \\
\hline Óbito & 46 & 3,3 & & & \\
\hline Alta & 1342 & 96,7 & & & \\
\hline Transferência & 4 & 0,3 & & & \\
\hline Tempo entre Coleta e Alta & & & & 4 & $2-7$ \\
\hline
\end{tabular}

Legenda: $\mathrm{n}$ - frequência absoluta. \% - frequência relativa percentual. IIQ - Intervalo Interquartil. PO - Pós-operatório. HAS - Hipertensão Arterial Sistêmica. UTI - Unidade de Terapia Intensiva. Fonte: Dados do estudo (2021).

$\mathrm{Na}$ Tabela 2, ao avaliar as características dos pacientes, observa-se que a presença de comorbidades $(\mathrm{p}<0,001)$ e de mialgia $(\mathrm{p}<0,001)$ e dor abdominal $(\mathrm{p}=0,002)$ como manifestação clínica estavam associadas à faixa etária, nesse caso aos adolescentes. Enquanto que dispneia $(\mathrm{p}=0,010)$ foi um sintoma mais frequente nas crianças. 
Tabela 2: Avaliação das características dos casos suspeitos de infecção pelo SARS-CoV-2 em um hospital de Sergipe de acordo com a faixa etária.

\begin{tabular}{|c|c|c|c|}
\hline & & a Etária & \\
\hline & Crianças & Adolescentes & p-valor \\
\hline Sexo, $n(\%)$ & & & \\
\hline Feminino & $577(44,6)$ & $50(53,2)$ & $0,106^{\mathrm{F}}$ \\
\hline Masculino & $717(55,4)$ & $44(46,8)$ & \\
\hline Procedência, $n(\%)$ & & & \\
\hline Capital & $1099(84,9)$ & $70(74,5)$ & $0,046^{\mathrm{Q}}$ \\
\hline Interior & $190(14,7)$ & $24(25,5)$ & \\
\hline Comorbidades, $n(\%)$ & $123(9,5)$ & $22(23,4)$ & $<0,001^{\mathrm{F}}$ \\
\hline HAS, $n(\%)$ & $49(39,8)$ & $12(54,5)$ & $0,198^{\mathrm{F}}$ \\
\hline Doença Cardiovascular, $n(\%)$ & $11(8,9)$ & $1(4,5)$ & $0,490^{\mathrm{F}}$ \\
\hline Desnutrição, $n(\%)$ & $3(2,4)$ & $0(0)$ & $0,459^{\mathrm{F}}$ \\
\hline Doenças cromossômicas, $n(\%)$ & $5(4,1)$ & $0(0)$ & $0,336^{\mathrm{F}}$ \\
\hline Doença Renal, $n(\%)$ & $2(1,6)$ & $0(0)$ & $0,547^{\mathrm{F}}$ \\
\hline Imunossupressão, $n(\%)$ & $6(4,9)$ & $0(0)$ & $0,290^{\mathrm{F}}$ \\
\hline Doença Metabólica, $n$ (\%) & $5(4,1)$ & $4(18,2)$ & $0,011^{\mathrm{F}}$ \\
\hline Doença Neurológica, $n(\%)$ & $10(8,1)$ & $3(13,6)$ & $0,405^{\mathrm{F}}$ \\
\hline Síndrome Genética, $n(\%)$ & $2(1,6)$ & $0(0)$ & $0,547^{\mathrm{F}}$ \\
\hline Doença Respiratória, $n$ (\%) & $67(54,5)$ & $12(54,5)$ & $0,995^{\mathrm{F}}$ \\
\hline Gestante de Risco, $n(\%)$ & $1(0,8)$ & $3(13,6)$ & $0,001^{\mathrm{F}}$ \\
\hline Laringomalacia, $n(\%)$ & $2(1,6)$ & $0(0)$ & $0,547^{\mathrm{F}}$ \\
\hline Obesidade, $n(\%)$ & $3(2,4)$ & $1(4,5)$ & $0,578^{\mathrm{F}}$ \\
\hline Outras Síndromes, $n(\%)$ & $3(2,4)$ & $0(0)$ & $0,459^{\mathrm{F}}$ \\
\hline PO de abdome obstrutivo, $n(\%)$ & $1(0,8)$ & $0(0)$ & $0,671^{\mathrm{F}}$ \\
\hline Prematuridade, $n(\%)$ & $5(4,1)$ & $0(0)$ & $0,336^{\mathrm{F}}$ \\
\hline Tumor, $n(\%)$ & $1(0,8)$ & $0(0)$ & $0,671^{\mathrm{F}}$ \\
\hline PCR Detectável, $n(\%)$ & $709(98,9)$ & $52(100)$ & $0,444^{\mathrm{F}}$ \\
\hline Tipo de Leito & & & \\
\hline Clínico, $n(\%)$ & $937(72,4)$ & $62(66)$ & $0,179^{\mathrm{F}}$ \\
\hline UTI, $n(\%)$ & $151(11,7)$ & $13(13,8)$ & $0,531^{\mathrm{F}}$ \\
\hline Estabilização, $n(\%)$ & $43(3,3)$ & $2(2,1)$ & $0,528^{\mathrm{F}}$ \\
\hline Ventilação Mecânica, $n$ (\%) & $63(4,9)$ & $5(5,3)$ & $0,845^{\mathrm{F}}$ \\
\hline Sintomático, $n(\%)$ & $1276(98,6)$ & $93(98,9)$ & $0,792^{\mathrm{F}}$ \\
\hline Cansaço, $n(\%)$ & $136(10,5)$ & $8(8,6)$ & $0,559^{\mathrm{F}}$ \\
\hline Cefaleia, $n(\%)$ & $2(0,2)$ & $0(0)$ & $0,704^{\mathrm{F}}$ \\
\hline Cianose, $n(\%)$ & $2(0,2)$ & $0(0)$ & $0,704^{\mathrm{F}}$ \\
\hline Corisa, $n(\%)$ & $319(24,7)$ & $28(30,1)$ & $0,242^{\mathrm{F}}$ \\
\hline Congestão Nasal, $n(\%)$ & $31(2,4)$ & $1(1,1)$ & $0,412^{\mathrm{F}}$ \\
\hline Desconforto Respiratório, $n(\%)$ & $309(23,9)$ & $16(17,2)$ & $0,141^{\mathrm{F}}$ \\
\hline Diarreia, $n(\%)$ & $65(5)$ & $4(4,3)$ & $0,756^{\mathrm{F}}$ \\
\hline Dispneia, $n(\%)$ & $478(37)$ & $22(23,7)$ & $0,010^{\mathrm{F}}$ \\
\hline Dor abdominal, $n(\%)$ & $16(1,2)$ & $5(5,4)$ & $0,002^{\mathrm{F}}$ \\
\hline Dor de Garganta, $n(\%)$ & $25(1,9)$ & $2(2,2)$ & $0,884^{\mathrm{F}}$ \\
\hline Espirro, $n(\%)$ & $320(24,7)$ & $17(18,3)$ & $0,160^{\mathrm{F}}$ \\
\hline Fadiga, $n(\%)$ & $7(0,5)$ & $1(1,1)$ & $0,512^{\mathrm{F}}$ \\
\hline Febre, $n(\%)$ & $851(65,8)$ & $52(55,9)$ & $0,053^{\mathrm{F}}$ \\
\hline Mialgia, $n(\%)$ & $0(0)$ & $1(1,1)$ & $<0,001^{\mathrm{F}}$ \\
\hline Saturação $\mathrm{O}_{2}<95 \%, n(\%)$ & $84(6,5)$ & $9(9,7)$ & $0,236^{\mathrm{F}}$ \\
\hline Taquicardia, $n(\%)$ & $3(0,2)$ & $0(0)$ & $0,642^{\mathrm{F}}$ \\
\hline Taquipneia, $n(\%)$ & $6(0,5)$ & $0(0)$ & $0,510^{\mathrm{F}}$ \\
\hline Tosse, $n(\%)$ & $993(76,8)$ & $79(84,9)$ & $0,070^{\mathrm{F}}$ \\
\hline Vômito, $n(\%)$ & $72(5,6)$ & $6(6,5)$ & $0,721^{\mathrm{F}}$ \\
\hline Tempo entre Coleta e Alta, Mediana (IIQ) & $4(2-8)$ & $3(2-7)$ & $0,022^{\mathrm{M}}$ \\
\hline
\end{tabular}

Legenda: $\mathrm{n}$ - frequência absoluta. \% - frequência relativa percentual. IIQ - Intervalo Interquartil. F - Teste Exato de Fisher. M - Teste de Mann-Whitney. PO - Pós-operatório. HAS - Hipertensão Arterial Sistêmica. UTI - Unidade de Terapia Intensiva. Fonte: Dados do estudo (2021).

Referente ao quadro clínico, a principal queixa foi tosse, relatada por 1.072 (77,2\%) pacientes, desses 993 (76,8\%) eram crianças e $79(84,9 \%)$ eram adolescentes, seguida por febre, a qual foi manifestação em $903(65,1 \%)$ casos, $851(65,8 \%)$ crianças e $52(55,9 \%)$ adolescentes. Destaca-se que $325(23,4 \%)$ pacientes relataram desconforto respiratório, mas apenas 93 $(6,7 \%)$ apresentaram saturação de $\mathrm{O}_{2}<95 \%$. Ademais, há outros sintomas mencionados em menor frequência como cefaleia, 
mialgia, vômito e dor abdominal.

Tabela 3: Comparação entre as características dos casos confirmados e excluídos de COVID-19 em um hospital de Sergipe.

\begin{tabular}{|c|c|c|c|}
\hline & \multicolumn{2}{|c|}{ Diagnóstico } & \multirow[b]{2}{*}{ p-valor } \\
\hline & COVID 19 & Outros & \\
\hline Idade, Mediana (IIQ) & $0,5(0,08-2)$ & $0,92(0,17-3)$ & $0,005^{\mathrm{M}}$ \\
\hline \multicolumn{4}{|l|}{ Faixa etária, $n(\%)$} \\
\hline Criança & $357(93,9)$ & $934(93,1)$ & \multirow[t]{2}{*}{$0,582^{\mathrm{F}}$} \\
\hline Adolescente & $23(6,1)$ & $69(6,9)$ & \\
\hline \multicolumn{4}{|l|}{ Sexo, $n(\%)$} \\
\hline Feminino & $177(46,6)$ & $449(44,8)$ & \multirow[t]{2}{*}{$0,545^{Q}$} \\
\hline Masculino & $203(53,4)$ & $554(55,2)$ & \\
\hline \multicolumn{4}{|l|}{ Procedência, $n(\%)$} \\
\hline Capital & $323(85)$ & $841(83,8)$ & \multirow[t]{2}{*}{$0,178^{Q}$} \\
\hline Interior & $54(14,2)$ & $160(16)$ & \\
\hline Comorbidades, $n(\%)$ & $45(11,8)$ & $100(10)$ & $0,310^{\mathrm{F}}$ \\
\hline HAS, $n(\%)$ & $16(35,6)$ & $45(45)$ & $0,287^{\mathrm{F}}$ \\
\hline Doença Cardiovascular, $n(\%)$ & $5(11,1)$ & $7(7)$ & $0,406^{\mathrm{F}}$ \\
\hline Desnutrição, $n(\%)$ & $1(2,2)$ & $2(2)$ & $0,931^{\mathrm{F}}$ \\
\hline Doenças cromossômicas, $n(\%)$ & $3(6,7)$ & $2(2)$ & $0,154^{\mathrm{F}}$ \\
\hline Doença Renal, $n(\%)$ & $0(0)$ & $2(2)$ & $0,339^{\mathrm{F}}$ \\
\hline Imunossupressão, $n(\%)$ & $3(6,7)$ & $3(3)$ & $0,305^{\mathrm{F}}$ \\
\hline Doença Metabólica, $n(\%)$ & $3(6,7)$ & $6(6)$ & $0,878^{\mathrm{F}}$ \\
\hline Doença Neurológica, $n(\%)$ & $5(11,1)$ & $8(8)$ & $0,544^{\mathrm{F}}$ \\
\hline Síndrome Genética, $n(\%)$ & $0(0)$ & $2(2)$ & $0,339^{\mathrm{F}}$ \\
\hline Doença Respiratória, $n(\%)$ & $23(51,1)$ & $56(56)$ & $0,584^{\mathrm{F}}$ \\
\hline Gestante de Risco, $n(\%)$ & $0(0)$ & $4(4)$ & $0,174^{\mathrm{F}}$ \\
\hline Laringomalacia, $n(\%)$ & $1(2,2)$ & $1(1)$ & $0,559^{\mathrm{F}}$ \\
\hline Obesidade, $n(\%)$ & $1(2,2)$ & $3(3)$ & $0,791^{\mathrm{F}}$ \\
\hline Outras Síndromes, $n(\%)$ & $1(2,2)$ & $2(2)$ & $0,931^{\mathrm{F}}$ \\
\hline PO de abdome obstrutivo, $n(\%)$ & $0(0)$ & $1(1)$ & $0,501^{\mathrm{F}}$ \\
\hline Prematuridade, $n(\%)$ & $2(4,4)$ & $3(3)$ & $0,659^{\mathrm{F}}$ \\
\hline Tumor, $n(\%)$ & $1(2,2)$ & $0(0)$ & $0,135^{\mathrm{F}}$ \\
\hline PCR Detectável, $n(\%)$ & $326(100)$ & $430(98,2)$ & $0,014^{\mathrm{F}}$ \\
\hline Tempo entre Coleta e Alta, Mediana (IIQ) & $5(3-10)$ & $4(2-7)$ & $<0,001^{M}$ \\
\hline
\end{tabular}

Legenda: $\mathrm{n}$ - frequência absoluta. \% - frequência relativa percentual. Q - Teste Qui-Quadrado de Pearson. F - Teste Exato de Fisher. M - Teste de MannWhitney. HAS - Hipertensão Arterial Sistêmica. PO - Pós-operatório. Fonte: Dados do estudo (2021).

A partir da Tabela 3 observa-se que o único fator associado ao diagnóstico de COVID-19 (p<0,001) é o intervalo entre o tempo da coleta do exame PCR-RT (Reação de Transcriptase Reversa Seguida de Reação em Cadeia da Polimerase) e da alta hospitalar. Ao comparar os casos confirmados de infecção por SARS-CoV-2 e os descartados, não houve diferença significativa no sexo, na procedência e na presença de comorbidades. 
Tabela 4: Comparação entre as características clínica e terapêuticas e o desfecho dos casos confirmados e excluídos de COVID-19 em um hospital de Sergipe.

\begin{tabular}{|c|c|c|c|}
\hline & \multicolumn{2}{|c|}{ Diagnóstico } & \multirow[b]{2}{*}{ p-valor } \\
\hline & COVID 19 & Outros & \\
\hline Sintomático, $n(\%)$ & $365(96,1)$ & $999(99,6)$ & $<0,001^{\mathrm{F}}$ \\
\hline Cansaço, $n(\%)$ & $27(7,1)$ & $117(11,7)$ & $0,014^{\mathrm{F}}$ \\
\hline Cefaleia, $n(\%)$ & $2(0,5)$ & $0(0)$ & $0,021^{\mathrm{F}}$ \\
\hline Cianose, $n(\%)$ & $0(0)$ & $2(0,2)$ & $0,385^{\mathrm{F}}$ \\
\hline Coriza, $n(\%)$ & $72(19)$ & $275(27,4)$ & $0,001^{Q}$ \\
\hline Congestão Nasal, $n(\%)$ & $12(3,2)$ & $20(2)$ & $0,194^{\mathrm{F}}$ \\
\hline Desconforto Respiratório, $n(\%)$ & $121(32)$ & $204(20,3)$ & $<0,001 \mathrm{Q}$ \\
\hline Diarreia, $n(\%)$ & $30(7,9)$ & $39(3,9)$ & $0,002^{\mathrm{F}}$ \\
\hline Dispneia, $n(\%)$ & $168(44,4)$ & $327(32,6)$ & $<0,001 \mathrm{Q}$ \\
\hline Dor abdominal, $n(\%)$ & $8(2,1)$ & $13(1,3)$ & $0,267^{\mathrm{F}}$ \\
\hline Dor de Garganta, $n(\%)$ & $10(2,6)$ & $17(1,7)$ & $0,255^{\mathrm{F}}$ \\
\hline Espirro, $n(\%)$ & $125(33,1)$ & $212(21,1)$ & $<0,001 \mathrm{Q}$ \\
\hline Fadiga, $n(\%)$ & $3(0,8)$ & $5(0,5)$ & $0,519^{\mathrm{F}}$ \\
\hline Febre, $n(\%)$ & $217(57,4)$ & $681(67,9)$ & $<0,001 \mathrm{Q}$ \\
\hline Mialgia, $n(\%)$ & $0(0)$ & $1(0,1)$ & $0,539^{\mathrm{F}}$ \\
\hline Saturação $\mathrm{O}_{2}<95 \%, n(\%)$ & $43(11,4)$ & $50(5)$ & $<0,001^{\mathrm{F}}$ \\
\hline Taquicardia, $n(\%)$ & $1(0,3)$ & $2(0,2)$ & $0,817^{\mathrm{F}}$ \\
\hline Taquipneia, $n(\%)$ & $0(0)$ & $6(0,6)$ & $0,132^{\mathrm{F}}$ \\
\hline Tosse, $n(\%)$ & $265(70,1)$ & $802(80)$ & $<0,001 \mathrm{Q}$ \\
\hline Vômito, $n(\%)$ & $21(5,6)$ & $57(5,7)$ & $0,927^{\mathrm{F}}$ \\
\hline \multicolumn{4}{|l|}{ Tipo de Leito } \\
\hline Clínico, $n(\%)$ & $280(73,7)$ & $715(71,3)$ & $0,376^{\mathrm{Q}}$ \\
\hline UTI, $n(\%)$ & $69(18,2)$ & $94(9,4)$ & $<0,001^{\mathrm{F}}$ \\
\hline Estabilização, $n(\%)$ & $14(3,7)$ & $31(3,1)$ & $0,579^{\mathrm{F}}$ \\
\hline Ventilação Mecânica, $n$ (\%) & $30(7,9)$ & $38(3,8)$ & $0,002^{\mathrm{F}}$ \\
\hline Óbito, $n(\%)$ & $23(6,1)$ & $23(2,3)$ & $0,001^{\mathrm{F}}$ \\
\hline
\end{tabular}

Legenda: $\mathrm{n}$ - frequência absoluta. \% - frequência relativa percentual. Q - Teste Qui-Quadrado de Pearson. F - Teste Exato de Fisher. UTI Unidade de Terapia Intensiva. Fonte: Dados do estudo (2021).

Através da Tabela 4 nota-se que as queixas de cefaleia $(p=0,021)$, diarreia $(p=0,002)$, desconforto respiratório $(\mathrm{p}<0,001)$, dispneia $(\mathrm{p}<0,001)$, espirro $(\mathrm{p}<0,001)$ e saturação de $\mathrm{O}_{2}<95 \%(\mathrm{p}<0,001)$ estavam relacionadas ao quadro de infecção pelo SARS-CoV-2. Assim como, a necessidade de uso de ventilação mecânica $(p=0,002)$, internação em UTI $(\mathrm{p}<0,001)$ e óbitos $(0,001)$. 
Tabela 5: Comparação entre as características dos casos suspeitos de COVID-19 que evoluíram para óbito em um hospital de Sergipe.

\begin{tabular}{|c|c|c|c|}
\hline & & & \\
\hline & Sim & Não & p-valor \\
\hline Idade, Mediana $(I I Q)$ & $0,08(0,05-0,58)$ & $0,83(0,17-3)$ & $<0,001^{M}$ \\
\hline Faixa etária, $n(\%)$ & & & \\
\hline Criança & $43(93,5)$ & $1251(93,2)$ & $0,945^{\mathrm{F}}$ \\
\hline Adolescente & $3(6,5)$ & $91(6,8)$ & \\
\hline Sexo, $n(\%)$ & & & \\
\hline Feminino & $19(41,3)$ & $608(45,3)$ & $0,592^{\mathrm{F}}$ \\
\hline Masculino & $27(58,7)$ & $734(54,7)$ & \\
\hline Procedência, $n(\%)$ & & & \\
\hline Capital & $27(58,7)$ & $1142(85,1)$ & $0,001 \mathrm{Q}$ \\
\hline Interior & $19(41,3)$ & $195(14,5)$ & \\
\hline Comorbidades, $n(\%)$ & $12(26,1)$ & $133(9,9)$ & $<0,001^{\mathrm{F}}$ \\
\hline PCR Detectável, $n(\%)$ & $35(100)$ & $726(98,9)$ & $0,535^{\mathrm{F}}$ \\
\hline Tipo de Leito & & & \\
\hline Clínico, $n(\%)$ & $10(21,7)$ & $989(73,7)$ & $<0,001^{\mathrm{F}}$ \\
\hline UTI, $n(\%)$ & $24(52,2)$ & $140(10,4)$ & $<0,001^{\mathrm{F}}$ \\
\hline Estabilização, $n(\%)$ & $9(19,6)$ & $36(2,7)$ & $<0,001^{\mathrm{F}}$ \\
\hline Ventilação Mecânica, $n$ (\%) & $22(47,8)$ & $46(3,4)$ & $<0,001^{\mathrm{F}}$ \\
\hline Sintomático, $n(\%)$ & $44(95,7)$ & $1325(98,7)$ & $0,077^{\mathrm{F}}$ \\
\hline Tempo entre Coleta e Alta, Mediana (IIQ) & $7(1-23)$ & $4(2-7)$ & $0,356^{\mathrm{M}}$ \\
\hline
\end{tabular}

Legenda: $\mathrm{n}$ - frequência absoluta. \% - frequência relativa percentual. IIQ - Intervalo Interquartil. F - Teste Exato de Fisher. M - Teste de Mann-Whitney. Fonte: Dados do estudo (2021).

Nota-se, a partir da Tabela 5, que houve uma relação entre os pacientes que evoluíram para óbito e a presença de comorbidades $(\mathrm{p}<0,001)$, como doenças cardiovasculares e cromossômicas $(\mathrm{p}<0,001)$, imunossupressão ( $\mathrm{p}<0,001)$, síndrome genética $(\mathrm{p}=0,031)$, doença renal $(\mathrm{p}=0,031)$ e neurológica $(\mathrm{p}=0,002)$. Além disso, alguns sintomas também estavam associados com o desfecho de óbito, como desconforto respiratório $(\mathrm{p}<0,001)$, espirro $(\mathrm{p}<0,001)$, taquicardia $(\mathrm{p}=0,004)$ e vômitos $(\mathrm{p}=0,026)$. Outros fatores relacionados com a evolução para óbito dos pacientes, foram a necessidade de internação em estabilização $(\mathrm{p}<0,001)$ e UTI $(\mathrm{p}<0,001)$.

\section{Discussão}

A prevalência da infecção pelo SARS-CoV-2 concentra-se em idosos e em adultos conforme a descrição encontrada na literatura, embora possa ocorrer em qualquer faixa etária. Na pediatria, encontramos diversos estudos como os de Dong Y Et al. e de Wang Y Et al. que trazem uma mediana de 7 anos para a prevalência da doença na faixa pediátrica. Já Hoang A Et al. descreveram uma mediana de 8,9 anos, enquanto o Centro de Controle de Doenças (CDC) dos Estados Unidos observou uma mediana de 11 anos. No Brasil, um estudo realizado em São Paulo revela uma mediana de 7 anos (Hoang et al., 2020). Embora a mediana seja variável de acordo com o estudo, percebe-se que a maioria aponta para a prevalência de crianças sobre os adolescentes, assim como foi demonstrado no presente estudo, além disso, na faixa etária pediátrica nota-se uma relevância do contágio intradomiciliar (FIOCRUZ et al., 2021).

No que tange ao sexo, este estudo identificou o masculino sendo o de maior prevalência tanto para os casos suspeitos (54,8\%), quanto para os casos confirmados $(53,4 \%)$ e também nos óbitos $(58,7 \%)$ pela COVID-19. Entretanto, ainda não há, na literatura, um consenso no que diz respeito à prevalência dessa doença em algum sexo, de modo que há diversos estudos que trazem a prevalência no sexo feminino, assim como há estudos que demonstram a prevalência no sexo masculino como, por exemplo, os dados presentes na Semana Epidemiológica 29 no Brasil (Escosteguy et al., 2021; MS 2019; Suleyman 2020).

O estudo de Escosteguy CC Et al., realizado em um Hospital Federal do Rio de Janeiro, com uma amostra de 356 pacientes com suspeita de COVID-19, variando de menores de 1 ano até 94 anos, relatou a presença de comorbidades na 
maioria dos casos $(80,1 \%)$, inclusive nos pacientes que possuíam até 19 anos $(15,2 \%)$, dentre as quais se destacaram doenças cardiovasculares $(40,2 \%)$ e diabetes mellitus $(25,3 \%)$. No presente estudo, que teve como amostra apenas a faixa etária até 19 anos, nota-se um padrão diferente associado à infecção pelo SARS-CoV-2, no qual predominam as doenças respiratórias como comorbidade. Isso pode ocorrer porque, devido às suas características típicas da anatomia, fisiologia e imunologia, as crianças são mais propensas a desenvolver doenças respiratórias (Bonfim et al., 2011; Procianoy et al., 2020).

Dentre os sintomas apresentados pelos pacientes pediátricos durante a infecção pelo SARS-CoV-2, o CDC (2020) dividiu a prevalência em dois grupos de acordo com a idade, nos quais, ainda que as manifestações clínicas se repitam, a frequência se diferencia entre elas. De 0 até 9 anos, há predomínio da febre (46\%), tosse (37\%), cefaleia (15\%) e diarreia (14\%). Já dos 10 aos 19 anos, tem-se cefaleia (42\%), tosse (41\%), febre (35\%) e mialgia (30\%) como manifestações mais prevalentes. Desse padrão, pode-se observar que nesta pesquisa não foi relatada mialgia por crianças, mas essa queixa aparece nos adolescentes ( $p<0,001)$, além disso a presença de comorbidades $(p<0,001)$ e dor abdominal $(p=0,002)$ também estava associado a essa faixa etária. Enquanto que a dispneia $(\mathrm{p}=0,010)$ foi um sintoma relacionado as crianças, apesar não ter sido relatada como sintoma mais frequente pelo CDC.

No estudo em questão, 145 crianças encontravam-se com alguma comorbidade, sendo que apenas foram 45 diagnosticadas com COVID-19. Diversos estudos demonstraram um cenário semelhante, como o de Hrusak O Et al., em que investigaram 200 crianças em tratamento oncológico e nove contraíram COVID-19, oito com sintomas leves e uma assintomática, o que sugere que a doença, em coexistência de câncer, não ocasiona aumento dos casos. No entanto, a presença de comorbidades na faixa etária pediátrica é vista como fator de risco, pois deve-se considerar o número de crianças sintomáticas com comorbidades que necessitam de ventilação mecânica ou evoluem para o óbito, como observado neste estudo, em que 12 crianças das 45 diagnosticadas com COVID-19 faleceram (Hrusak et al., 2020; Yamamoto et al., 2020).

Ademais, sabe-se que é de suma importância que seja realizado um diagnóstico em casos pediátricos, seja pela possibilidade de evolução para comorbidades ou casos graves, seja por as crianças serem possíveis facilitadoras da transmissão viral (Souza et al., 2020). Desta maneira, o CDC recomenda que sejam testados os pacientes com base na presença de sinais e sintomas compatíveis com COVID-19, enfatizando a febre e os sintomas respiratórios nos critérios de casos suspeitos (CDC, 2020). De forma semelhante, a presente pesquisa observou o sintoma de febre em $65,1 \%$ das crianças, além da alta prevalência de sintomas respiratórios, como a tosse $(77,2 \%)$, sendo esta a principal queixa encontrada, além de desconforto respiratório $(23,4 \%)$, o que atende aos critérios para casos suspeitos. No entanto, é válido salientar que as crianças podem não ser testadas com tanta frequência por, muitas vezes, apresentarem-se oligossintomáticas ou assintomáticas, levando a uma subestimativa do número real de pessoas infectadas e ao aumento da transmissão do vírus (Souza et al., 2020).

A pesquisa em questão demonstrou, também, que o diagnóstico de COVID-19 foi confirmado em 399 (28,7\%) casos, sendo que, dos casos descartados, 1.003 (72,3\%) apresentaram diagnóstico por outras etiologias e 5 (0,4\%) tiveram diagnóstico etiológico de infecção pelo vírus Influenza. Desta forma, é importante ressaltar que, em caso de identificação de outros patógenos respiratórios, a pesquisa para SARS-CoV-2 ainda deve ser realizada, pois não se deve excluir uma possível infecção, como observado no estudo de Kim D et al, em que 20,7\% das 116 amostras positivas para SARS-CoV-2 foram positivas para um ou mais patógenos adicionais (Souza et al., 2020; CDC, 2020).

Neste estudo, 18,2\% dos pacientes diagnosticados com COVID-19 apresentaram critérios para internamento em Unidade de Terapia Intensiva (UTI), 7,9\% com necessidade de ventilação mecânica, sendo que 6,1\% dos casos evoluíram para o óbito. As crianças admitidas em UTI podem se apresentar como SRAG, não SRAG ou Síndrome Inflamatória Multissistêmica da Criança (MIS-C). É válido salientar que a SRAG acomete, principalmente, a faixa etária até 1 ano de idade na pediatria (FIOCRUZ, 2020). Até a Semana Epidemiológica 29 já haviam sido notificados, em pacientes de 0 a 19 anos, 4.670 casos de hospitalização de SRAG com diagnostico de COVID-19 com a evolução para óbito de 541 desses pacientes 
(MS, 2019). A falta de evidências de alta qualidade assim como de consenso entre os autores se encontram como barreiras no conhecimento sobre a infecção pelo SARS-CoV-2 e a SRAG nesses pacientes neonatais gerando questionamentos sobre como e quando deve ser realizada a assistência respiratória desse paciente (Procianoy et al., 2020; Kim et al., 2020).

Por fim, a literatura evidencia que as crianças raramente apresentam formas graves da doença, consequentemente, tendo menos casos que evoluem para óbito. Desta forma, esta pesquisa observou que houve uma relação entre os pacientes que evoluíram para óbito e a presença de comorbidades $(p<0,001)$ e necessidade de internação em estabilização (p<0,001) e UTI $(\mathrm{p}<0,001)$. Um estudo semelhante nos Estados Unidos também ressaltou este fato, em que, na amostra de pacientes pediátricos admitidos em unidades de terapia intensiva com diagnóstico de COVID-19, 83\% apresentavam comorbidades pré-existentes, $38 \%$ necessitaram de ventilação invasiva e $23 \%$ apresentaram falência de 2 ou mais sistemas orgânicos, sendo que, deste total, $4 \%$ veio a óbito (Silva et al., 2020; Shekerdemian et al., 2020).

\section{Conclusão}

Faz-se notório que, em relação aos demais grupos etários, ainda há uma escassez de informações na literatura acerca da COVID-19 em crianças e em adolescentes. Ademais, a maioria dos casos pediátricos são assintomáticos ou leves, sendo enfatizado, também no estudo, que as crianças apresentam melhor evolução na COVID-19, com o pequeno percentual que evolui a óbito geralmente associado com a presença de comorbidades pré-hospitalares, que é um fator importante para a gravidade da infecção. Isto ressalta a importância de trabalhos como este, pois traz aspectos epidemiológicos dessa faixa etária que poderão ajudar na compreensão dessa doença em tal grupo o que fomenta a promoção de medidas preventivas mais efetivas. A principal limitação desse estudo foi o fato de não conseguir realizar o diagnóstico etiológico dos casos que não foram confirmados para infecção pelo SARS-CoV-2, além de não avaliar a terapêutica instituída para os pacientes com COVID-19, apesar de que estes pontos podem ser trabalhados por estudos futuros. A principal contribuição desse estudo é que a maioria dos casos suspeitos não foram confirmados, tratando-se de outras infecções virais que são comuns nessa faixa etária.

\section{Agradecimentos}

Gostaria de agradecer ao Programa de Bolsas de Iniciação Científica da Universidade Tiradentes (PROBIC/Unit) pelo financiamento do projeto. Além de todo subsídio do Hospital e Maternidade Santa Isabel para coleta de dados.

\section{Referências}

Aydogdu, A. L. F. (2020). Saúde mental das crianças durante a pandemia causada pelo novo coronavírus: revisão integrativa. J Health NPEPS.5(2). https://doi.org/10.30681/252610104891

Bonfim, C. M., Nogueira, M. L., Simas, P.V., Gardinassi, L. G., Durigon, E. L., Rahal, P. \& Souza, F. P. (2011). Frequent respiratory pathogens of respiratory tract infections in children attending daycare centers. J Pediatr. 87(5):439-44. http://dx.doi.org/10.2223/JPED.2126

Centro de Controle de Doenças (CDC). (2020). COVID-19 RESPONSE TEAM. Coronavirus Disease 2019 in Children - United States, 2020. MMRV Morb Mortal Wkly Rep. 69 (14): 422-426.

CDC Health Alert Network. Updated guidance on evaluating and testing persons for coronavirus disease 2019 (COVID-19). USA, 2020. https://emergency.cdc.gov/han/2020/HAN00429.asp

Deville, J. G., Song., E. \& Ouellette, C. P. (2021). COVID-19 manifestações clínicas e diagnóstico em crianças, 2021. In UpToDate. https://www.uptodate.com/contents/covid-19-clinical-manifestations-and-diagnosis-in-

children?search=covid $\% 2019 \% 20 \mathrm{e} \% 20 \mathrm{crianca} \&$ source=search_result\&selectedTitle=1 150\&usage_type=default\&display_rank=1.

Dong, Y., Mo, X., Hu, Y., Qi, X., Jiang, F., Jiang, Z. \& Tong, S. (2021) Epidemiology of COVID-19 among children in china. Pediatrics. 145(6). https://doi.org/10.1097/INF.0000000000002739

Escosteguy, C. C., Eleuterio, T. A., Pereira, A. G. L., Marques, M. R. V. E., Brandão, A. D. \& Batista, J. P. M. (2021). COVID-19: estudo seccional de casos suspeitos internados em um hospital federal do Rio de Janeiro e fatores associados ao óbito hospitalar. Epidemiologia e Serviços de Saúde. $30(1)$ : e2020750. http://dx.doi.org/10.1590/s1679-49742021000100023 
Fundação Oswaldo Cruz (FIOCRUZ). Instituto Nacional de Saúde da Mulher, da Criança e do Adolescente Fernandes Figueira. (2020). COVID-19 e Saúde da Criança e do Adolescente. https://portaldeboaspraticas.iff.fiocruz.br/atencaocria nca/covid-19-saude-crianca-e-adolescente.

Hillesheim, D., Tomasi, Y. T., Figueiró, T. H., Paiva, K. M. (2020). Síndrome respiratória aguda grave por COVID-19 em crianças e adolescentes no Brasil: perfil dos óbitos e letalidade hospitalar até a 38 Semana Epidemiológica de 2020. Epidemiologia e Serviços de Saúde. 29 (5):e2020644. https://doi.org/10.1590/S1679-49742020000500021

Hoang, A., Chorath, K., Moreira, A., Evans, M., Burmeister-Morton, F., Burmeister, F., Naqvi, R., Petershack, M. \& Moreira, A. (2020). COVID-19 in 7780 pediatric patients: a systematic review. Eclinical Medicine. 4 (1): 100433. https://doi.org/10.1016/j.eclinm.2020.100433

Hrusak, O., Kalina, T., Wolf, J., Balduzzi, A., Provenzi, M., Rizzari, C., Rives, S., Del Pozo Carlavilla, M., Alonso, M. E. V., Domínguez-Pinilla, N., Bourquin, J. P., Schmiegelow, K., Attarbaschi, A., Grillner, P., Mellgren, K., van der Werff Ten Bosch, J., Pieters, R., Brozou, T, ..., Schrappe, M. (2020). Flash survey on severe acute respiratory syndrome Coronavirus-2 infections in paediatric patients on anticancer treatment. Eur J Cancer.132:11-16. https://doi.org/10.1016/j.ejca.2020.03.021

Kim, D., Quinn, J., Pinsky, B., Shah, N. H. \& Brown. I. (2020). Rates of co-infection between SARS-CoV-2 and other respiratory pathogens. JAMA. 323(20):2085-2086. https://doi.org/10.1001/jama.2020.6266

Lima, E. A. R., Lima, M. A. C., Costa, T. A., Leite, D. C. F., Oliveira, H. F., Azevedo, M. R. D. \& Porto, C. P. S. (2021). Covid-19 suspicious cases in pediatrics: an analysis in a tertiary hospital of Brazil. International Journal of Development Research. 11(04): 45908-45912. https://doi.org/10.37118/ijdr.21463.04.2021

Lu, X., Zhang, L., Du, H., Zhang, J., Li, Y. Y., Qu, J., Zhang, W., Wang, Y., Bao, S., Li, Y., Wu, C., Liu, H., Liu, D., Shao, J., Peng, X., Yang, Y., Liu, Z., Xiang, Y., Zhang, F., Silva, R. M., ... Wong, G. W. K. (2020). SARS-CoV-2 infection in children. New Engl J Med. 382(17):663-5. https://doi.org/10.1056/NEJMc2005073

Martins, L.A., Santos, D. V., Marques, P. F., Silva, E. A. L., Castro, C. T., Santos, D. B. \& Camargo, C. L. (2021). Quadro clínico da população pediátrica com SARS-CoV-2 e cuidados: revisão. Rev Gaúcha Enferm. 42(esp):e20200162. https://doi.org/10.1590/1983-1447.2021.20200162

Ministério da Saúde (BR). Secretaria de Vigilância em Saúde. (2020). Doença pelo coronavírus COVID-19. Bol Epidemiol. 32. https://www.gov.br/saude/ptbr/assuntos/boletins-epidemiologicos-1/set/boletim-epidemiologico-covid-32-final-23-09_18h30.pdf.

Ministério da Saúde (BR). Secretaria de Vigilância em Saúde. (2020). Especial: doença pelo coronavírus 2019. Bol Epidemiol. https://saude.gov.br/ima ges/pdf/2020/July/22/Boletim-epidemiologico-COVID-23-final.pdf.

Procianoy, R. S., Silveira, R. C., Manzoni, P. \& Sant'Anna, G. (2020). Neonatal COVID-19: little evidence and the need for more information. J Pediatr. 96:269-72. https://doi.org/10.1016/j.jped.2020.04.002

Qiu, H., Wu, J., Hong, L., Luo, Y., Song, Q. \& Chen, D. (2020). Clinical and epidemiological features of 36 children with coronavirus disease 2019 (COVID19) in Zhejiang, China: an observational cohort study. Lancet Infect Dis.20(6):689-96. https://doi.org/10.1016/S1473-3099(20)30198-5

Ramos, R. T., Silva, B. C. C., Araújo, G. C. B., Riedi, C. A., Ibiapina, C. C., Bezerra, P. G. M., Ribeiro, J. D. \& Santana, M. F. P. (2020). Aspectos respiratórios da COVID-19 na infância: o que o pediatra precisa saber? Residência Pediátrica. 10:2. https://doi.org/10.25060/residpediatr-2020.v10n2-349

Shekerdemian, L. S., Mahmood, N. R. \& Wolfe, K. K. (2020). Characteristics and Outcomes of Children With Coronavirus Disease 2019 (COVID-19) Infection Admitted to US and Canadian Pediatric Intensive Care Units. JAMA Pediatrics. 174(9):868-873. https://doi.org/10.1001/jamapediatrics.2020.1948

Silva, J. R. A., Argentino, A. C. A., Dulaba, L. D., Bernardelli, R. R. \& Campiolo, E. L. (2020). COVID-19 em pediatria: um panorama entre incidência e mortalidade. Resid Pediatr. 10(3):1-4. https://doi.org/10.25060/residpediatr-2020.v10n3-383

Souza, T. H., Nadal, J. A., Nogueira, R. J. N., Pereira, R. M. \& Brandão, M. B. (2020). Clinical manifestations of children with COVID-19: A systematic review. Pediatr Pulmonol.55(8):1892-1899. https://doi.org/10.1002/ppul.24885

Suleyman, G., Fadel, R. A., Malette, K. M., Hamond, C., Abdulla, H., Entz, A., Demertzis, Z., Hanna, Z., Failla, A., Dagher, C., Chaudhry, Z., Vahia, A., Lanfranco, O. A., Ramesh, M., Zervos, M. J., Alangaden, G., Miller, J. \& Brar, I. (2020). Clinical characteristics and morbidity associated with coronavirus disease 2019 in a Series of patients in metropolitan Detroit. JAMA Netw Open.3(6):e2012270. http://dx.doi.org/10.1001/jamanetworkopen.2020.12270

Toso, B. R. G. O, Viera, C. S., Furtado, M. C. C. \& Bonati, P. C. R. (2020). Ações de Enfermagem no cuidado à criança na atenção primária durante a pandemia de COVID-19. Rev Soc Bras Enferm Pediatr.20(spe):6-15. https://doi.org/10.31508/1676-3793202000000122

Wang Y, Zhu F, Wang C, Wu J, Liu J, Chen X. et al. (2020). Children hospitalized with Severe COVID-19 in Wuhan. Pediatr Infect Dis J. 39 (7): 91 - 94. https://doi.org/10.1542/peds.2020-0702

Yamamoto, L., Santos, E. H. D., Pinto, L. S., Rocha, M. C., Kanunfre, K. A., Vallada, M. G. \& Okay, T. S. (2020). SARS-CoV-2 infections with emphasis on pediatric patients: a narrative review. Rev Inst Med Trop Sao Paulo. 4;62: e65. https://doi.org/10.1590/S1678-9946202062065 